identified in a large proportion of the tumors across the distinct $\mathrm{COO}$ subtypes (Figure 1). Applying the publicly available LymphGen algorithm on the GOYA dataset could help classify patients into well-defined molecularly and clinically distinct subgroups. These newly characterized subsets can identify patients with an unfavorable prognosis and may guide the development of new precision therapies for these aggressive lymphomas.

\section{Funding}

Funded by a grant from LympheCo.

\section{References}

1. Alizadeh $\mathrm{AA}$, Eisen $\mathrm{MB}$, Davis RE, et al. Distinct types of diffuse large B-cell lymphoma identified by gene expression profiling. Nature. 2000;403(6769):503-511.

2. Pasqualucci L, Trifonov V, Fabbri G, et al. Analysis of the coding genome of diffuse large B-cell lymphoma. Nat Genet. 2011;43(9):830-837.

3. Lohr JG, Stojanov P, Lawrence MS, et al. Discovery and prioritization of somatic mutations in diffuse large B-cell lymphoma (DLBCL) by whole-exome sequencing. Proc Natl Acad Sci U S A. 2012;109(10):3879-3884

4. Rosenwald A, Wright G, Chan WC, et al. The use of molecular profiling to predict survival after chemotherapy for diffuse large-B-cell lymphoma. N Engl J Med. 2002;346(25):1937-1947

5. Bolen CR, Klanova M, Trneny M, et al. Prognostic impact of somatic mutations in diffuse large B-cell lymphoma and relationship to cellof-origin: data from the phase III GOYA study. Haematologica. 2020;105(9):2298-2307.

6. Schuetz JM, Johnson NA, Morin RD, et al. BCL2 mutations in diffuse large B-cell lymphoma. Leukemia. 2012;26(6):1383-1390.

7. Seymour JF, Kipps TJ, Eichhorst B, et al. Venetoclax-Rituximab in Relapsed or Refractory Chronic Lymphocytic Leukemia. N Engl J Med. 2018;378(12):1107-1120.

8. Morschhauser F, Feugier P, Flinn IW, et al. Venetoclax plus rituximab, cyclophosphamide, doxorubicin, vincristine and prednisolone (R$\mathrm{CHOP}$ ) improves outcomes in BCL2-positive first-line diffuse large B-cell lymphoma (DLBCL): first safety, efficacy and biomarker analyses from the phase II CAVALLI study. Blood. 2018;132 (Supplement 1):782.
9. Morschhauser F, Salles G, McKay P, et al. Interim Report from a Phase 2 Multicenter Study of Tazemetostat, an EZH2 Inhibitor: Clinical Activity and Favorable Safety in Patients with Relapsed or Refractory B-Cell Non-Hodgkin Lymphoma. Clin Lymphoma Myeloma Leuk. 2017;17(Suppl 2):S380-381.

10. Assouline SE, Nielsen TH, Yu S, et al. Phase 2 study of panobinostat with or without rituximab in relapsed diffuse large B-cell lymphoma. Blood. 2016;128(2):185-194.

11. Ying CY, Dominguez-Sola D, Fabi M, et al. MEF2B mutations lead to deregulated expression of the oncogene BCL6 in diffuse large B cell lymphoma. Nat Immunol. 2013;14(10):1084-1092.

12. Schmitz R, Wright GW, Huang DW, et al. Genetics and Pathogenesis of Diffuse Large B-Cell Lymphoma. N Engl J Med. 2018;378 (15):1396-1407.

13. Chapuy B, Stewart C, Dunford AJ, et al. Molecular subtypes of diffuse large B cell lymphoma are associated with distinct pathogenic mechanisms and outcomes. Nat Med. 2018;24(5):679-690.

14. Wright GW, Huang DW, Phelan JD, et al. A Probabilistic Classification Tool for Genetic Subtypes of Diffuse Large B Cell Lymphoma with Therapeutic Implications. Cancer Cell. 2020;37 (4):551-568.e14.

15. Vermaat JS, Somers SF, de Wreede LC, et al. MYD88 mutations identify a molecular subgroup of diffuse large B-cell lymphoma with an unfavorable prognosis. Haematologica. 2020;105(2):424-434.

16. Kraan W, Horlings HM, van Keimpema M, et al. High prevalence of oncogenic MYD88 and CD79B mutations in diffuse large B-cell lymphomas presenting at immune-privileged sites. Blood Cancer J. 2013;3(9):e139

17. Yonese I, Takase H, Yoshimori M, et al. CD79B mutations in primary vitreoretinal lymphoma: Diagnostic and prognostic potential. Eur J Haematol. 2019;102(2):191-196.

18. Schrader AMR, Jansen PM, Willemze R, et al. High prevalence of MYD88 and CD79B mutations in intravascular large B-cell lymphoma. Blood. 2018;131(18):2086-2089.

19. Kraan W, van Keimpema M, Horlings HM, et al. High prevalence of oncogenic MYD88 and CD79B mutations in primary testicular diffuse large B-cell lymphoma. Leukemia. 2014;28(3):719-720.

20. Wilson WH, Young RM, Schmitz R, et al. Targeting B cell receptor signaling with ibrutinib in diffuse large B cell lymphoma. Nat Med. 2015;21(8):922-926.

21. Lionakis MS, Dunleavy K, Roschewski M, et al. Inhibition of B Cell Receptor Signaling by Ibrutinib in Primary CNS Lymphoma. Cancer Cell. 2017;31(6):833-843.e5.

22. Grommes C, Pastore A, Palaskas N, et al. Ibrutinib Unmasks Critical Role of Bruton Tyrosine Kinase in Primary CNS Lymphoma. Cancer Discov. 2017;7(9):1018-1029.

\title{
Thrombin generation: a global coagulation procedure to investigate hypo- and hyper-coagulability
}

\author{
Armando Tripodi \\ IRCCS Ca' Granda Maggiore Hospital Foundation, Angelo Bianchi Bonomi Hemophilia and Thrombosis Center and Fondazione \\ Luigi Villa, Milano, Italy \\ E-mail: ARMANDO TRIPODI - armando.tripodi@unimi.it
}

doi:10.3324/haematol.2020.253047

$\mathrm{T}$ he article by van Paridon et al. ${ }^{1}$ published in this issue of Haematologica on results of thrombin generation (TG) in cardiovascular disease and mortality, stemming from the Gutenberg Health Study, provides an opportunity to comment on TG as a global laboratory procedure to investigate hypo- and hyper-coagulability.

TG as a laboratory test was developed in the early 1950s by McFarlane and Biggs ${ }^{2}$ and was based on the activation of coagulation in whole blood or plasma by triggers such as tissue factor or cephaline and calcium chlo- ride. The amount of thrombin generated over time was titrated by sampling the mixture at different time points into a fibrinogen solution and the resultant clotting times interpolated from a dose-response calibration curve to derive thrombin concentrations. Years later, Hemker et al. made substantial changes..$^{3-5}$ The fibrinogen solution was replaced by a chromogenic substrate specific for thrombin, test plasma was defibrinated prior to testing and computer software was developed to derive the parameters stemming from the TG curve. These changes made 


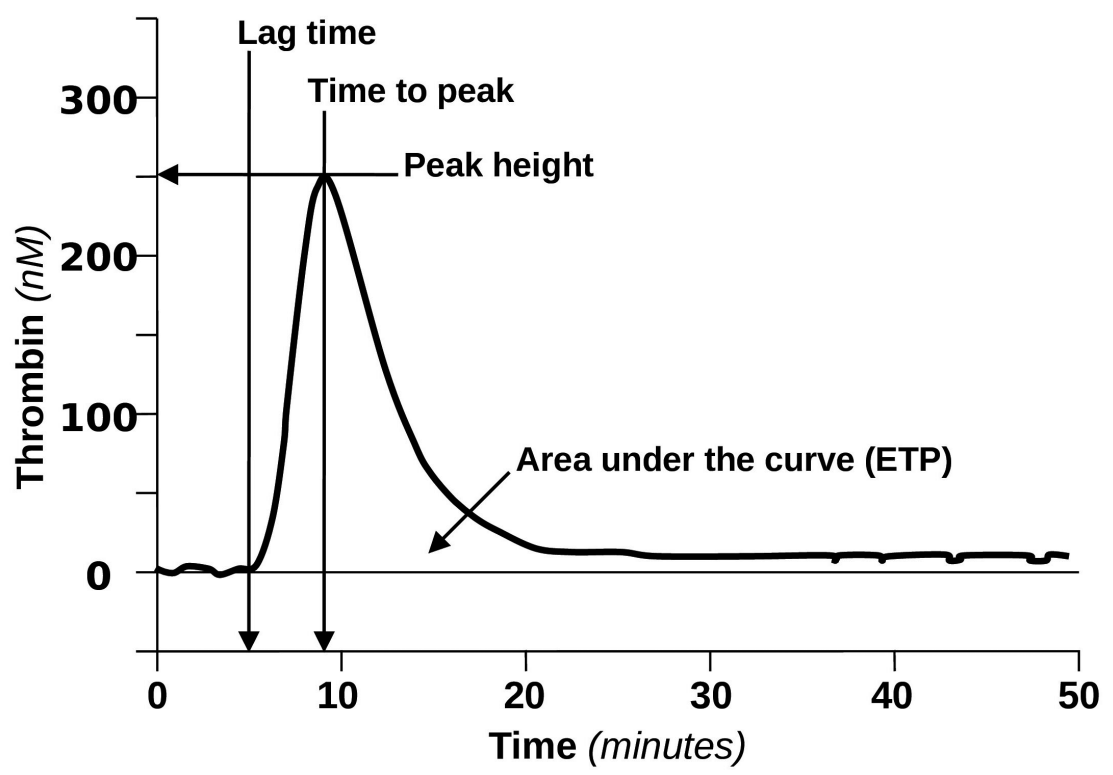

Figure 1. Typical thrombin generation curve with related parameters that can be obtained after in vitro activation of coagulation in plasma. See text for more explanation. ETP: endogenous thrombin potential.

TG much easier to perform even in less specialized laboratories. In the early 1990s TG was further modified to include a slow-acting chromogenic substrate that allowed thrombin to be monitored continuously, avoiding the subsampling procedures. More recently, the chromogenic substrate has been replaced by a fluorogenic one, which obviates plasma defibrination and makes TG applicable to platelet-poor or platelet-rich plasma. Currently, TG is designed to operate on a microtiter plate-based fluorimeter, which, in combination with the computer software, displays TG curves and calculates the parameters illustrated in Figure 1: the lag-time, peak thrombin concentration, time to reach peak concentration and the endogenous thrombin potential (ETP). The lag-time is defined as the time needed for the first amounts of thrombin to be generated; it can be regarded as the conventional plasma clotting time and is expected to decrease or increase in conditions associated with hyper- or hypo-coagulability, respectively. The peak-thrombin defines the highest thrombin concentration that can be obtained under the experimental conditions and is expected to increase or decrease in conditions associated with hyper- or hypocoagulability, respectively. The time to reach the peak defines the velocity of TG and should be prolonged or shortened in conditions associated with hypo- or hypercoagulability, respectively. The area under the curve, known as the ETP, represents the net amount of thrombin that the test plasma can generate under the experimental conditions. ETP is considered to be the resultant of the two opposing drivers operating in plasma that favor TG (procoagulants) and thrombin decay (anticoagulants). Accordingly, when performed under standardized conditions, TG can be considered a laboratory tool suitable for investigating hypo- and hyper-coagulability much better than the conventional global tests of coagulation, such as the time-honored prothrombin time (PT) and activated partial thromboplastin time (APTT). PT and APTT are in fact static tests in which plasma clots soon after tiny amounts of thrombin (5\%) are produced, leaving the remaining part undetected. ${ }^{6}$ Furthermore, PT and APTT are responsive to procoagulant factors (which is why they are used to diagnose hemophilia and allied disorders), but they are much less responsive to naturally occurring anticoagulants (which is why they are normal rather than shortened in patients with congenital deficiency of protein $\mathrm{C}$ or antithrombin).

It is therefore unsurprising that over the last two decades, TG attracted the attention of many researchers. Currently, there are 7,446 reports under the term "thrombin generation" published in PubMed and most deal with the application of TG to investigate hypo- or hyper-coagulability. A scrutiny of the reported manuscripts reveals that TG has been applied to five categories: (i) to help elucidate the mechanisms of thrombogenesis in clinical conditions for which precise knowledge is still poor; (ii) laboratory diagnosis of disorders of hemostasis; (iii) monitoring treatment with pro-hemostatic agents; (iv) monitoring treatment with antithrombotic drugs; and (v) predicting the risk of recurrence of venous thromboembolism. The following paragraphs summarize the current state-of-the-art concerning the application of TG testing.

\section{Elucidation of mechanisms of thrombogenesis}

This application has been the most productive in terms of results achieved. For example, the TG procedure when performed as such or modified by the addition of thrombomodulin has been instrumental in challenging the old dogma of liver cirrhosis as the prototype of acquired hemorrhagic coagulopathies. ${ }^{7,8} \mathrm{TG}$ has also been useful for understanding the mechanisms of thrombogenesis in a variety of clinical conditions associated with increased risk of thrombosis (especially venous thrombosis) such as obesity, ${ }^{9}$ diabetes, ${ }^{10}$ and Cushing disease. ${ }^{11}$

\section{Laboratory diagnosis of hemostatic disorders}

The TG procedure has been useful for understanding the contribution of the plasma levels of the individual coagulation factors in determining the bleeding pheno- 
type in subjects with hemophilia ${ }^{12}$ or other rare congenital diseases. $^{13}$

\section{Monitoring treatment with pro-hemostatic agents}

In principle, every treatment aimed at preventing/treating hemorrhage accomplishes its goal by increasing thrombin generation. In this respect, the TG procedure is the most promising laboratory tool to monitor patients on treatment. As an example, TG has been used in hemophilia with inhibitors to factor VIII or IX to tailor treatment with conventional bypassing agents. ${ }^{14}$ These drugs achieve their hemostatic effect with no substantial modification of factors VIII/IX, thus making the measurement of these factors after infusion practically useless. However, the studies carried out so far, while providing evidence that TG is increased according to the dose of the drug infused to treat/prevent hemorrhage, failed to provide conclusive evidence that the laboratory endpoint is associated with clinical outcomes. ${ }^{15}$ Therefore, the procedure is not yet approved by the regulatory authorities for routine use in hemophilia patients being treated with bypassing agents.

\section{Monitoring treatment with antithrombotic drugs}

There is no doubt that TG is decreased in a dose-dependent fashion in patients treated with any antithrombotic drug (e.g., heparins, parenteral direct thrombin inhibitors, warfarin or direct oral anticoagulants). However, there is still no conclusive evidence that the TG procedure is superior for treatment monitoring to the APTT (unfractionated heparin), anti-factor Xa assays (low molecular weight heparin), the PT-International Normalized Ratio (warfarin) or the specific measurement of the plasma concentration of direct oral anticoagulants.

\section{Predicting the risk of recurrent venous thromboembolism}

There is evidence stemming from clinical trials that the amount of thrombin generated after the in-vitro activation of coagulation is a good predictor of the risk of a recurrence of venous thromboembolism. In this respect, TG should be equivalent (as a risk predictor) to D-dimer, ${ }^{16,17}$ which, following the seminal observations of Palareti and co-workers, ${ }^{18}$ is used to make decisions on the optimal duration of anticoagulation to prevent recurrence of venous thromboembolism. Although these studies showed that TG and D-dimer are independently associated with the risk of recurrent venous thromboembolism, there is no conclusive evidence that performing the two measurements simultaneously can improve risk prediction in individual patients. ${ }^{18}$

\section{New observations on thrombin generation}

The above observations are instrumental to supporting the old concept that hypercoagulability, when assessed by a global coagulation procedure, is one of the key mechanisms that explain the risk of thrombosis, the others being reduced blood flow and endothelial dysfunction (collectively known as Virchow triad). In this issue of the Journal van Paridon et al. add more evidence on the mechanisms of hypercoagulability and clinically relevant outcomes. ${ }^{1}$ They evaluated TG in a large population of subjects $(n=5,000)$ enrolled in a prospective study with a rel- atively long follow-up (up to 9.65 years). The authors found that some TG parameters were independently associated with overall mortality. In particular, the study showed that the ETP and lag-time were directly associated with overall mortality. Furthermore, these parameters were associated with some conditions (e.g., age, obesity, diabetes, dyslipidemia, use of oral contraceptives or hormonal replacement therapy) that are known risk factors for cardiovascular disease. The study by van Paridon et al. is the first to investigate the association of hypercoagulability (as measured by TG) and the risk of mortality in a large population. ${ }^{1}$ However, some issues emerge from the study that warrant consideration. While it is plausible that high ETP is directly associated with overall mortality, it is less plausible that the prolonged (not shortened) lagtime is associated with mortality. It should however be recognized that a prolonged lag-time has been hypothesized to be associated with increased levels of tissue factor pathway inhibitor, one of the naturally occurring anticoagulants. Unfortunately, the authors did not provide data on tissue factor pathway inhibitor in their cohort, nor did they report other parameters of TG such as the time to reach the peak concentration. One may wonder whether or not the latter parameter would be more representative than the lag-time to describe the real situation concerning the velocity of TG, which might help to understand the mechanism of thrombogenesis. Furthermore, the study did not investigate the association between TG and cardiovascular mortality. This would have been a more plausible association between hypercoagulability and clinical outcome. Notwithstanding these limitations, the study by van Paridon et al. should be considered an important step forward for the development of TG as a global procedure to assess the hemostatic system and its relationship with overall mortality. ${ }^{1}$

\section{References}

1. van Paridon PCS, Panova-Noeva M, van Oerle R, et al. Thrombin generation in cardiovascular disease and mortality - results from the Gutenberg Health Study. Haematologica. 2020;105(9):2327-2334.

2. Macfarlane RG, Biggs R. A thrombin generation test. J Clin Pathol. 1953;6(1):3-7

3. Hemker HC, Willems GM, Béguin S. A computer assisted method to obtain the prothrombin activation velocity in whole plasma independent of thrombin decay processes. Thromb Haemost. 1986;56(1):9-17.

4. Hemker HC, Wielders S, Kessels H, Béguin S. Continuous registration of thrombin generation in plasma, its use for the determination of the thrombin potential. Thromb Haemost .1993;70(4):617-624.

5. Hemker HC, Giesen PL, Ramjee M, et al. The thrombogram: monitoring thrombin generation in platelet-rich plasma. Thromb Haemost. 2000;83(4):589-591.

6. Mann KG, Brummel K, Butenas S. What is all that thrombin for? J Thromb Haemost. 2003;1(7):1504-1514.

7. Tripodi A, Salerno F, Chantarangkul V, et al. Evidence of normal thrombin generation in cirrhosis despite abnormal conventional coagulation tests. Hepatology. 2005;41(3):553-558.

8. Tripodi A, Mannucci PM. The coagulopathy of chronic liver disease. N Engl J Med. 2011;365(2):147-156.

9. Tripodi A, Primignani M, Badiali S, et al. Body mass index reduction improves the baseline procoagulant imbalance of obese subjects. J Thromb Thrombolysis. 2019;48(1):52-60.

10. Tripodi A, Branchi A, Chantarangkul V, et al. Hypercoagulability in patients with type 2 diabetes mellitus detected by a thrombin generation assay. J Thromb Thrombolysis. 2011;31(2):165-172.

11. Tripodi A, Ammollo CT, Semeraro F, et al. Hypercoagulability in patients with Cushing disease detected by thrombin generation 
assay is associated with increased levels of neutrophil extracellular trap-related factors. Endocrine. 2017;56(2):298-307.

12. Santagostino E, Mancuso ME, Tripodi A, et al. Severe hemophilia with mild bleeding phenotype: molecular characterization and global coagulation profile. J Thromb Haemost. 2010;8(4):737-743.

13. Zekavat OR, Haghpanah S, Dehghani J, et al. Comparison of thrombin generation assay with conventional coagulation tests in evaluation of bleeding risk in patients with rare bleeding disorders. Clin Appl Thromb Hemost. 2014;20(6):637-644.

14. Tran HT, Sørensen B, Bjørnsen S, et al. Monitoring bypassing agent therapy - a prospective crossover study comparing thromboelastometry and thrombin generation assay. Haemophilia. 2015;21(2):275283.

15. Tripodi A, Chantarangkul V, Novembrino C, Peyvandi F. Advances in the treatment of hemophilia: implications for laboratory testing.
Clin Chem. 2019;65(2):254-262.

16. Tripodi A, Legnani C, Chantarangkul V, et al. High thrombin generation measured in the presence of thrombomodulin is associated with an increased risk of recurrent venous thromboembolism. J Thromb Haemost. 2008;6(8):1327-1333.

17. van Hylckama Vlieg A, Baglin CA, Luddington $\mathrm{R}$, et al. The risk of a first and a recurrent venous thrombosis associated with an elevated D-dimer level and an elevated thrombin potential: results of the THE-VTE study. J Thromb Haemost. 2015;13(9):1642-1652.

18. Palareti G, Cosmi B, Legnani C, et al. D-dimer testing to determine the duration of anticoagulation therapy. N Engl J Med. 2006;355 (17):1780-1789.

19. Eichinger S, Hron G, Kollars M, Kyrle PA. Prediction of recurrent venous thromboembolism by endogenous thrombin potential and D-dimer. Clin Chem. 2008;54(12):2042-2048. 